7. Salipante SJ, SenGupta DJ, Cummings LA, Land TA, Hoogestraat DR, Cookson BT. Application of whole-genome sequencing for bacterial strain typing in molecular epidemiology. J Clin Microbiol 2015;53:1072-1079.

\section{Bacteriophage M13 May Be Used for the Assessment of Viral Transfer during Doffing of Ebola-Level Personal Protective Equipment}

To the Editor-Extensive barrier precautions can prevent skin and mucous membrane contamination during the patient care. However, personal protective equipment (PPE) can be contaminated with body fluids and infectious virus after a patient care encounter. ${ }^{1,2}$ We read with interest the articles by Casanova et $\mathrm{al}^{3}$ and Kwon et $\mathrm{al}^{4}$, which reported a certain level of self-contamination with nonenveloped viruses and a much lower rate of self-contamination with enveloped viruses, with contamination limited to inner gloves. Recently, Casanova et $\mathrm{al}^{5}$ and Mumma et $\mathrm{al}^{6}$ (from the Centers for Disease Control and Prevention Epicenters Program, Division of Healthcare Quality Promotion, United States) further assessed the contamination of skin, gloves, and scrubs after doffing Ebolalevel PPE. In these studies, they assessed self-contamination risks using 2 surrogate viruses, bacteriophages MS2 and $\Phi 6$, to represent nonenveloped and enveloped viruses, respectively. However, given that both MS2 and $\Phi 6$ are spherical bacteriophages and are much smaller than the filamentous Ebola virus, their adhesion capabilities on PPE are much different. Thus, the reported contamination rates after doffing Ebolalevel PPE may be inaccurate.

Ebola virus is an enveloped RNA virus with a filamentous appearance and a uniform diameter of $\sim 80 \mathrm{~nm}$, but Ebola virus particles vary greatly in length. In general, the median particle length of Ebola viruses ranges from 974 to $1,086 \mathrm{~nm}$. $^{7}$ In contrast, bacteriophage $\Phi 6$ has a pleomorphic appearance and a uniform diameter of $\sim 80 \mathrm{~nm},{ }^{8}$ which is almost 10 times shorter than the average length of an Ebola virus particle (Figure 1). In microbial fermentations, small increases in hyphal length (eg, the formation of pellets or clumps) can cause large increases in broth viscosity because filamentous bioparticles have higher adhesion forces than spherical bioparticles. ${ }^{9}$ Thus, the adhesion capability of $\Phi 6$ on PPE may be much lower than that of Ebola virus. Thus, the bacteriophage $\Phi 6$ might not be an ideal surrogate virus.

In detailed studies, Casanova et $\mathrm{al}^{5}$ found that no $\Phi 6$ transfer to inner gloves, hands, or face among 10 healthcare workers. Only 1 healthcare worker had $\Phi 6$ on scrubs at low levels $\left(1.4 \times 10^{2}\right)$ This contamination rate was much lower than that of nonenveloped bacteriophage MS2: 2 healthcare worker had MS2 on scrubs, 1 had it on hands, and 7 had it on inner gloves (at $10^{1}-10^{6}$ ). Despite these differences, the fault

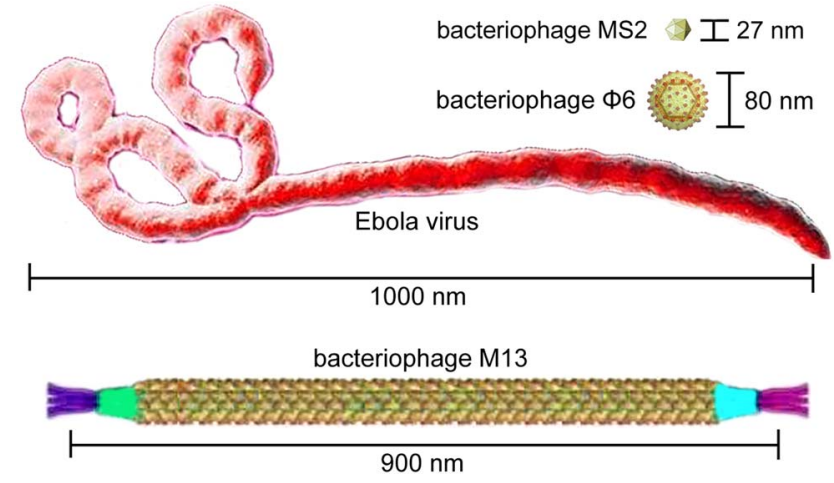

FIGURE 1. Lengths and shapes of Ebola virus and bacteriophages MS2, 66 , and M13.

trees for MS2 and $\Phi 6$ contamination suggested similar pathways. ${ }^{6}$ Similarly, very low levels of $\Phi 6$ contamination (much lower than those of MS2) have also been reported in previous studies. ${ }^{3,4}$ However, for the aforementioned reason, the risk the doffing protocol for Ebola-level PPE may be underestimated when $\Phi 6$ is selected as the surrogate virus. Also, the risk that the doffing protocol for Ebola-level PPE may be overestimated when MS2 is selected as the surrogate virus must be considered.

Different from $\Phi 6$ and MS2, M13 is a filamentous bacteriophage with a $900 \mathrm{~nm}$ particle length, ${ }^{10}$ which is very close to the average length of Ebola viruses (Figure 1). The M13 bacteriophage can be easily cultured and detected with the visible fluorescent marker, making it an ideal surrogate virus for the biocontainment study on the Ebola-level PPE. Presumably, a more accurate contamination rate after doffing Ebola-level PPE could be achieved using the surrogate virus M13. Nevertheless, we agree with the authors that the doffing protocols should be improved for better protections against all types of viruses, especially the filamentous Ebola viruses with high adhesion capabilities.

\section{ACKNOWLEDGMENTS}

Financial support: No financial support was provided relevant to this article. Potential conflicts of interest: All authors report no conflicts of interest relevant to this article.

\section{Shu Yuan, PhD; ${ }^{1}$ Zhong-Wei Zhang, PhD; ${ }^{1}$ Zi-Lin Li, $\mathrm{MD}^{2}$}

Affiliations: 1. College of Resources, Sichuan Agricultural University, Chengdu, China; 2. Xijing Hospital, Medical University of the Air Force, Xi'an, China.

Address correspondence to Shu Yuan, College of Resources, Sichuan Agricultural University, Chengdu 611130, China (roundtree318@hotmail.com). Infect Control Hosp Epidemiol 2018;39:762-763

(C) 2018 by The Society for Healthcare Epidemiology of America. All rights reserved. 0899-823X/2018/3906-0026. DOI: 10.1017/ice.2018.74 


\section{REFERENCES}

1. Tomas ME, Kundrapu S, Thota P, et al. Contamination of health care personnel during removal of personal protective equipment. JAMA Intern Med 2015;175:1904-1910.

2. Verbeek JH, Ijaz S, Mischke C, et al. Personal protective equipment for preventing highly infectious diseases due to exposure to contaminated body fluids in healthcare staff. Cochrane Database Syst Rev 2016;4:CD011621.

3. Casanova LM, Teal LJ, Sickbert-Bennett EE, et al. Assessment of selfcontamination during removal of personal protective equipment for Ebola patient care. Infect Control Hosp Epidemiol 2016;37:1156-1161.

4. Kwon JH, Burnham CD, Reske KA, et al. Assessment of healthcare worker protocol deviations and self-contamination during personal protective equipment donning and doffing. Infect Control Hosp Epidemiol 2017;38:1077-1183.

5. Casanova LM, Erukunuakpor K, Kraft CS, et al. Assessing viral transfer during doffing of Ebola-level personal protective equipment in a biocontainment unit. Clin Infect Dis 2018;66: 945-949.

6. Mumma JM, Durso FT, Ferguson AN, et al. Human factors risk analyses of a doffing protocol for Ebola-level personal protective equipment: mapping errors to contamination. Clin Infect Dis 2018;66:950-958.

7. Feldmann H, Klenk HD. Marburg and Ebola viruses. Adv Virus Res 1996;47:1-52.

8. Katz A, Alimova A, Futerman E, Katz G, Wei H, Gottlieb P. Bacteriophage $\varphi 6$-structure investigated by fluorescence stokes shift spectroscopy. Photochem Photobiol 2012;88:304-310.

9. Papagianni M. Fungal morphology and metabolite production in submerged mycelial processes. Biotechnol Adv 2004;22: 189-259.

10. Stopar D, Spruijt RB, Wolfs CJ, Hemminga MA. Protein-lipid interactions of bacteriophage M13 major coat protein. Biochim Biophys Acta 2003;1611:5-15. 